\title{
An Improved Actuated Signal Control of Intersection Based on VISVAP
}

\author{
Yaru Guo ${ }^{1, a}$ and Jihui $\mathrm{Ma}^{1,2, b}$ \\ ${ }^{1}$ School of Traffic and Transportation, Beijing Jiaotong University, Beijing 100044, China \\ ${ }^{2}$ MOE Key Laboratory for Urban Transportation Complex Systems Theory and Technology, Beijing \\ Jiaotong University, Beijing 100044, China \\ a14120770@bjtu.edu.cn, bjhma@bjtu.edu.cn
}

Keywords: Logic of actuated signal control; Traffic simulation; Signal control; VISVAP

\begin{abstract}
Traffic congestion has become an inevitable problem in modern society, and the signal control of intersection plays an important role to ensure the effective operation of urban transport. The paper proposes an improved actuated signal control model to improve the running efficiency of intersection. In order to verify the effectiveness of the improved algorithm, the paper uses VISSIM to simulate the operating condition of different durations under timing control, actuated signal control and improved actuated signal control based on actual traffic flow, signal timing and other data of intersection. The simulation result shows that travel time and delay time are both reduced under the control of actuated signal in different time periods. And the improved actuated signal control is more effective than other signal controls.
\end{abstract}

\section{Introduction}

At present, most of the intersections adopt fixed cycle signal control mode. The signal control mode cannot change phase according to actual traffic flow, that will increase the unnecessary waiting time of the vehicle when the traffic fluctuation greatly in some intersections. In order to improve the situation, people began to study the actuated signal control. Webester [1] proposed a delay model and the formal of green time for each phase, which established the foundation for actuated signal control. The same year, actuated signal control with detector and electronic computer with the function of the options appeared [2]. The inductive signal control mode, which greatly promoted the development of signal controlled intersections. Rouphail [3] proposed a delay model about actuated control, which is close to the actual delay in in case of low saturation. Francesco [4] proposed a probabilistic model for estimating the length of the next phase queue based on the analysis of the vehicle arrival probability. The paper can calculate the next phase of the effective green time through the model. Moreover, the actuated coordinated control of multi-intersection is optimized in the paper of Yun et al. [5]. And the paper realized bidirectional filter control. Jing et al. [6] researched vehicle arrival rate and analyzed probabilistic relations of traffic headway and unit green extension. Li et al. [7] used full-induction control method to solve the conflicts in urban arterial highway intersections. And results show that can effectively alleviates congestion. In view of the traffic management situation in China, point control is still one of the most important measures of control. In this paper, the logic control is improved. The paper adds queuing length and waiting time as condition for the conversion of phase.

\section{Actuated Signal Control}

Actuated Signal Control Parameter. To achieve actuated control, the following parameters need to be used.

1) The minimum green time $\left(G_{\min }\right)$

Each phase sets a period of minimum green time in the early time. Regardless of whether the phase or other phases has a car came, the phase must ensure the minimum green time. Setting minimum green time should consider several factors:

a) $G_{\min }$ should ensure vehicles that stop between detector and stop line can all out of stop line. 
b) $G_{\min }$ should ensure pedestrians can safely pass the street, we usually set it to7-13s.

2) Unit extension time $\left(G_{0}\right)$

In a certain time interval, if a car is detected after the minimum green time, the system will extend the green time, or that is judged to be the end of green time. Therefore, unit extension time, as an important parameter, can judge whether stop the flow of traffic. Unit extension time plays a decisive role for the efficiency of the actuated signal control. Setting unit extension time should consider several factors:

a) $G_{0}$ should ensure the vehicle can leave the stop line from the detector. The formula is as follows [2][4][8][9]:

$$
G_{0}=D / v
$$

where $D$ is the distance of detector to stop line, $v$ is the average speed of vehicle.

b) The proper length of the unit extension time should be as far as possible without the loss of green time. As long as vehicle interval shorter than unit extension time, the phase will always show green time. In order to improve traffic efficiency, the unit extension time should be as short as possible when the time meets the needs of actual transport, rather than waiting for not closely followed vehicle through the intersection.

c) In a phase, all individual detectors are usually associated, so the number of lanes must be noted when determining the unit extension time.

3) The maximum green time $\left(G_{\max }\right)$

$G_{\max }$ time is the limitation of green time in order to keep optimal green-time-rate. When the phase reaches the $G_{\max }$, the signal system will be forced closed, then other phase will change to green light. In fact, $G_{\max }$ is the signal time of optimal cycle and green-time-rate assigned to each phase of the green time, which is set to 30-60s.

4) Traffic flow (Q)

Traffic flow is formed from continuous vehicles which run on the road. The method of confirming traffic can take advantage of the real-time test which can directly take the traffic volume as the input of the control scheme optimization process.

5) Time headway (h)

The definition of headway is the time interval of a section of a vehicle queue of consecutive vehicles driving on the same lane, which plays an important role in actuated signal control and is a key factor to change the phase. Methods of manual counts and image method can be used to obtain the time headway. In addition, the time headway can be calculated by the average and saturation flow. The formula is as follows:

$$
\mathrm{h}=3600 / S
$$

where $S$ is saturation flow.

The Basic Principle of Actuated Control Model. The traffic actuated control system is composed of signal control and wagon detector. The timing parameters are the minimum green time, unit extension time, the maximum green time and the location of the detector (distance between detector and stop line) in the actuated control. Fig. 1 is the working principle of the actuated signal, which embodies the basic working principle of the actuated control. Actuated signal controller set $G_{\min }$ when using the phase. The phase will be changed to other phase, if no follow-up vehicle arrives. Otherwise, the green time will be extended. That is to say, if traffic flow was broken, the system would change phase in the present time interval, otherwise the green time would be extended [2]. No matter whether has a car arrives, the phase will be ended if the green time reaches the maximum green time. As can be seen from the above, $G_{\min } \leq G \leq G_{\max }$.

Improved Actuated Signal Control. VAP model, which is a module of VISVAP, can be used to write inductive programs after knowing the principle of actuated signal control. Actuated logic is the basis of the implementation of the actuated control. And it is a prerequisite for operating actuated control, in which each phase of the right passage and green time will be controlled by whether the detector detects the vehicle's arrival or the queue length of a certain phase. But general actuated control will select phase in sequence (if there are phase 1, phase 2 and phase 3 , the signal will be loop 
execution in the order of $1,2,3$ ) according to the working principle of actuated signal. The method cannot automatically select phase according to the weight of traffic demand. So this paper proposed a control method based on the waiting time and queue length. Specific induction control process steps are as follows:

Step 1: To determine whether green time is up to $G_{\min }$ in the current phase. If the conditions are met, it will go to step 2. Otherwise, keeping the current phase;

Step 2: To determine whether there is any vehicle to arrive in the period of $G_{0}$. If it is to keep the phase until green time ups to $G_{\max }$. Otherwise, going to step 3;

Step 3: The next phase is selected, which uses queue length and waiting time as constraint conditions. At the same time to detect whether waiting time and the vehicle's queuing are greater than the maximum values. If it is to change phase, otherwise go to step 4.

Step 4: To determine the next phase whether is the current phase. If the conditions are met, it will go to step 2.

The logic rules of actuated signal control are shown in Fig. 2:

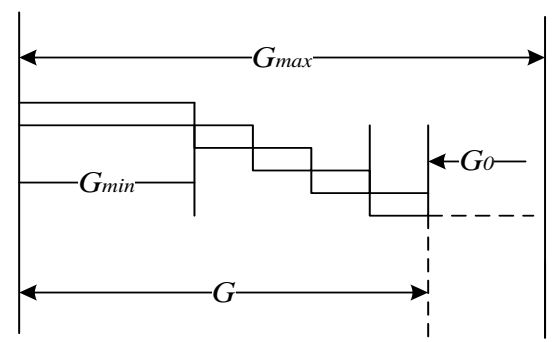

Figure 1. The Working Principle of Actuated Signal

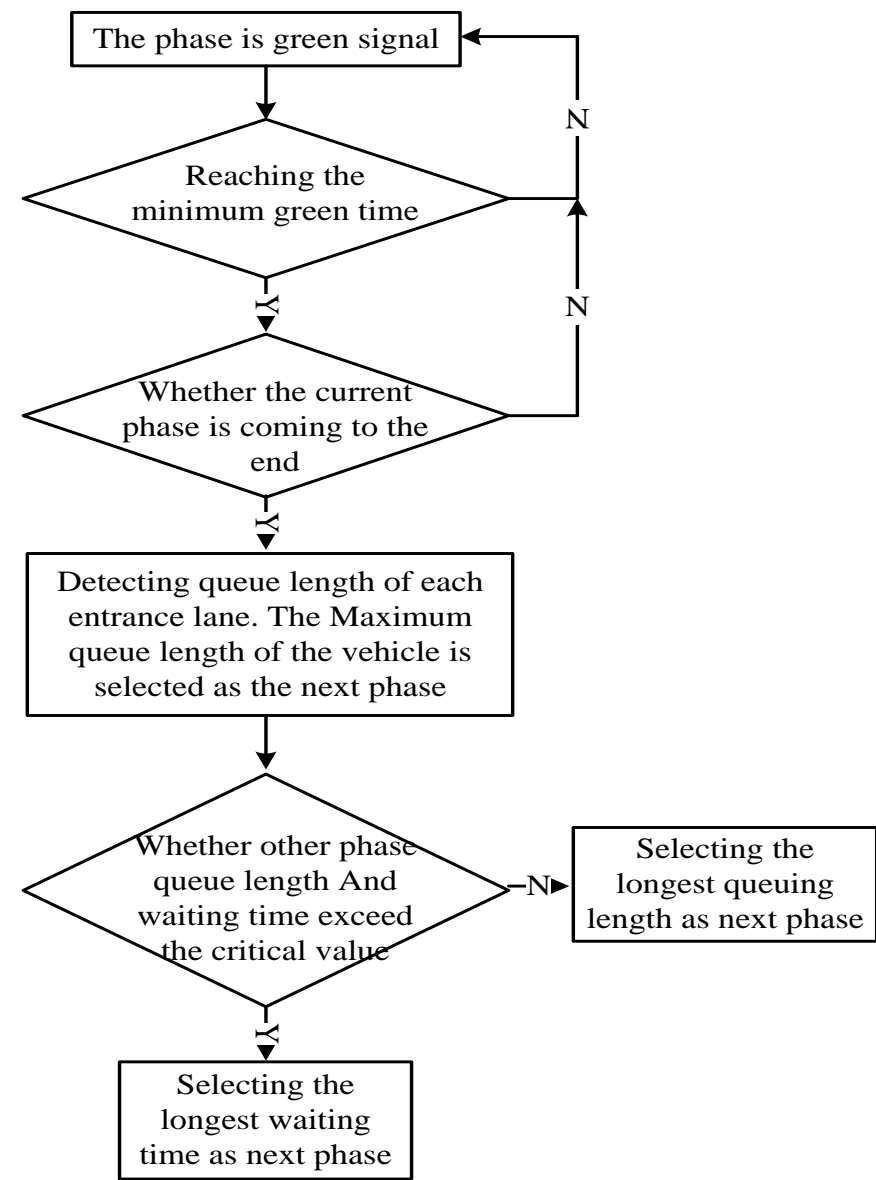

Figure 2. Logic Rules of Actuated Signal Control

The Evaluating Parameter of Signal Control Benefit. Effectiveness evaluation is not only to 
measure and evaluate the control system performance of some parameters or physical quantities, and is the objective function to make control strategy, optimize the control parameters [10]. Highway Capacity Manual 2000 (USA) uses average delay of vehicle as the only standard of service level. At the same time people feel the most direct is travel delay and travel time when they have a travel. So the evaluation indicators of this paper are delay and travel time. The delay reflects vehicle's blocked time and loss of travel time, etc. Delay is a comprehensive reflection of the intersection of the design and the evaluation index, which will be effected by composed of lane, cycle time, green-time-rate, saturation and other indicators. Travel time reflects the traffic condition of the vehicle's overall travel time, which is the important parameter of the traffic benefit. Reduced travel time can improve operating efficiency of traffic facilities and punctuality of bus, save the travel time of passenger; reduce the cost of public transportation management.

\section{Traffic Simulation}

VISSIM is a microscope traffic simulation system which can analyze traffic operation situation under the constraints of traffic signal and traffic composition and output all kinds of traffic evaluation parameters in the form of file. Therefore, it is an important tool to analyze and evaluate traffic applicability in the construction of transport infrastructure. In order to verify the validity of improved actuated control, the case simulated the operation of peak hour and hollow hour about one intersection. At the same time signal control phase of the intersection is shown in Fig. 3, and traffic flow is shown in Table 1. The meaning of 1-12 are as follows: $1,2,3$ respectively represent straight, left turn and right turn of west entrance lane; 4, 5, 6 respectively represent straight, left turn and right turn of south entrance lane; 7, 8, 9 respectively represent straight, left turn and right turn of east entrance lane; 10, 1112 respectively represent straight, left turn and right turn of north entrance lane.

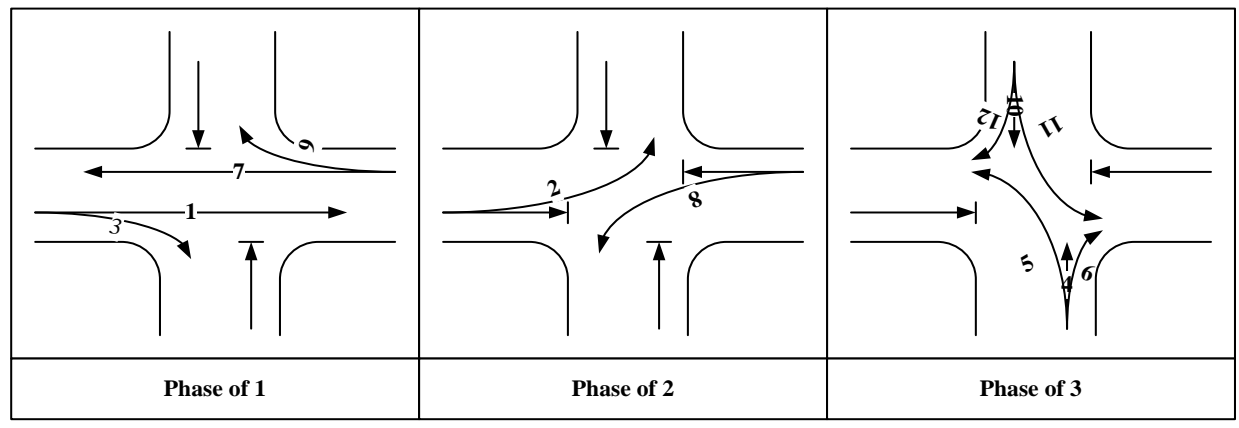

Figure 3. Signal Control Phase

Table 1 Traffic flow

$[\mathrm{pch} / \mathrm{h}]$

\begin{tabular}{c|c|cccccccccccc}
\hline & Period/s & 1 & 2 & 3 & 4 & 5 & 6 & 7 & 8 & 9 & 10 & 11 & 12 \\
\hline \multirow{4}{*}{ Peak } & $0 \sim 900$ & 814 & 244 & 80 & 554 & 164 & 240 & 1064 & 202 & 208 & 460 & 226 & 418 \\
hour & $901 \sim 1800$ & 764 & 252 & 136 & 486 & 152 & 196 & 1042 & 204 & 164 & 392 & 136 & 304 \\
& $1801 \sim 2700$ & 812 & 192 & 92 & 586 & 164 & 252 & 1058 & 156 & 178 & 432 & 188 & 284 \\
& $2701 \sim 3600$ & 748 & 192 & 132 & 548 & 152 & 296 & 954 & 166 & 190 & 380 & 240 & 240 \\
\hline \multirow{4}{*}{ Hollow } & $0 \sim 900$ & 568 & 86 & 40 & 388 & 80 & 136 & 750 & 132 & 100 & 352 & 92 & 80 \\
hour & $901 \sim 1800$ & 494 & 118 & 52 & 550 & 116 & 114 & 570 & 124 & 206 & 328 & 98 & 134 \\
& $1801 \sim 2700$ & 660 & 92 & 84 & 466 & 116 & 142 & 804 & 172 & 130 & 360 & 142 & 94 \\
& $2701 \sim 3600$ & 626 & 102 & 48 & 420 & 126 & 124 & 738 & 162 & 170 & 348 & 146 & 150 \\
\hline
\end{tabular}

In the process of the simulation, VAP model explains and creates the logic control commands. At the same time, all kinds of detectors collect traffic information in real time, and carry out logic processing in the process of simulation [11]. In order to better compare the simulation results, the paper also simulated timing signal control. The different signal controls of the peak hour and hollow 
hour are simulated by VISSIM, and the results are described by using line chart. The line chart is shown in Fig. 4

Compared with the line charts, the improved model can improve the efficiency of the intersection, especially when the traffic is at peak time. Compared with timing control, the average delay time dropped about $23 \%$ for actuated control and about $36 \%$ for improved actuated control in the period of hollow. The average travel time respectively reduced about $25 \%$ and $35 \%$. In the period of peak, the average delay time reduced about $1 \%$ and $34 \%$. The average travel time respectively reduced about $1 \%$ and $25 \%$. Through the comparison of the tested data, some conclusions are drawn that the improved model really improves the performance.

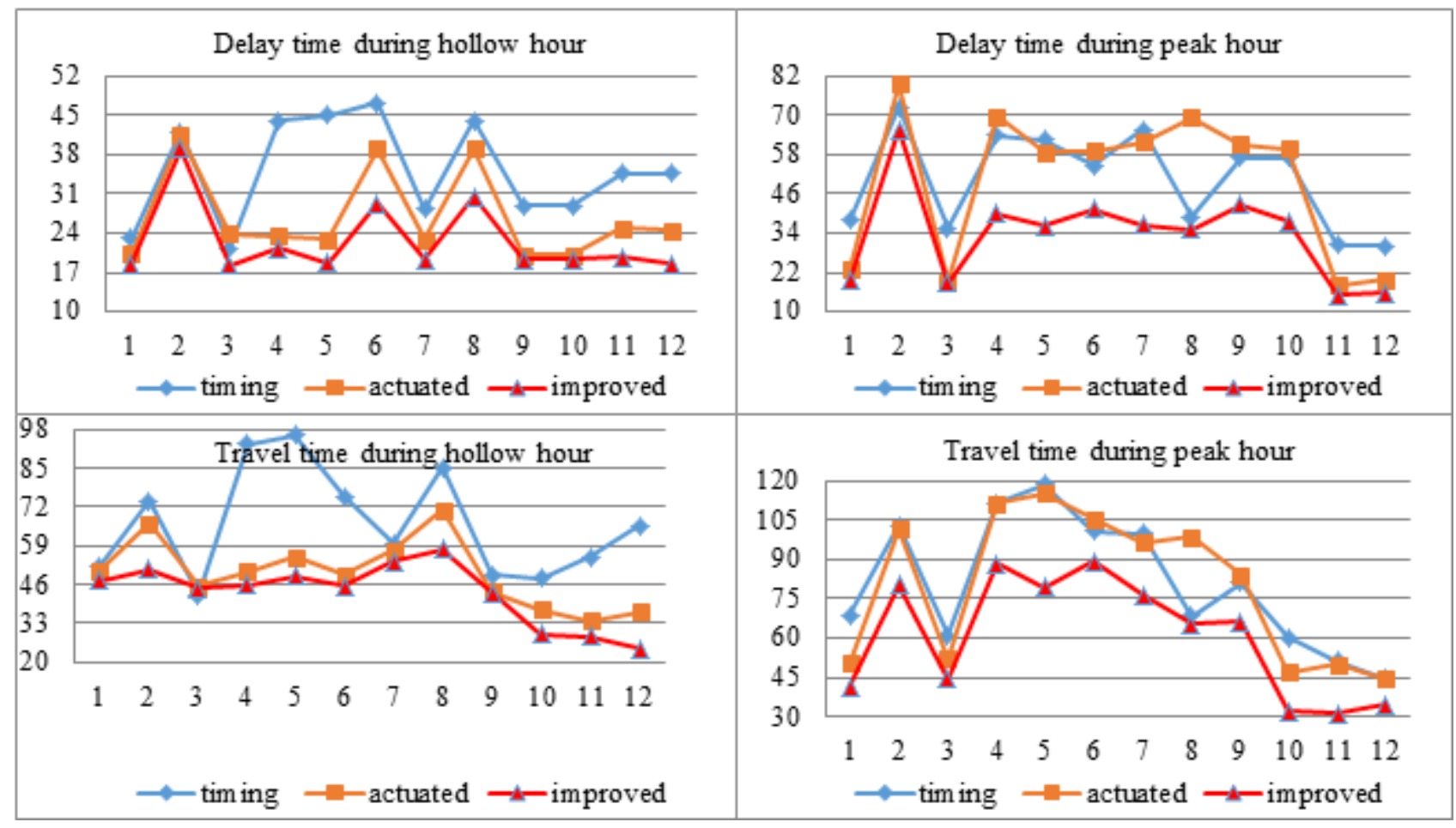

Figure 4. Comparison of results

\section{Summary}

In this paper, the waiting time and the queue length are used as the constraint conditions to select which phase has right of way. The weight of traffic demand is considered in the model. Finally the efficiency is verified by using VISSIM. The conclusion can be drawn that the improved actuated signal control not only can improve the efficiency, but also can efficiently improve the shortcoming of actuated control that cannot adapt to high saturation of traffic flow.

\section{References}

[1] Webster F V. Traffic signal settings [J]. Road Research Technical Paper, 1958, 39.

[2] Peikun Yang, Bing Wu. Traffic Management and Control. Beijing: China Communications Press, 2003.2.

[3] Rouphail N, Anwar M, Fambro D, et al. Validation of Generalized Delay Model for Vehicle-Actuated Traffic Signals [J]. Transportation Research Record Journal of the Transportation Research Board, 1997, 1572(1):105-111.

[4] Francoins Dion, Bruce Hellinga A Rule-based Real-time Traffic Responsive Signal Control System with Transit Priority. Application to an Isolated intersection Transportation Research Part B: Methodological, Vol.36B No.4 2002: 325-343. 
[5] Yun I, Park B. Stochastic Optimization for Coordinated Actuated Traffic Signal Systems[J]. Journal of Transportation Engineering, 2012, 138(7):819-829.

[6] Jing Tai, Lv Bin, Hao Binbin. Actuated Intersection Signal Control Using Probability Model[J]. Journal of Transport Information and Safety, 2014(1):16-20.

[7] Li J, Wang Y, Zhang Y, et al. Full-Induction Control Method of Urban Arterial Highway Intersections [J]. 2015.

[8] Hongbin Yin, S. C. wong, Jianmin Xu, C. K. Wong, Urban Traffic Flow Prediction Using a Fuzzy-neural Approach. Transportation Research PartC: Emerging Technologies Vol. 10C Issue.2 2002: 85-98

[9] Wei Huang, Lide Chen. Overview of Intelligent Transportation System (ITS). Beijing: China Communications Press, 1999, 16

[10]Qian Wang. REAEARCH OF URBAN TRAFFIC SIGNAL CONTROL SYSTEM ABSTRACT: [master thesis]. Shanghai: Tongji University, 1987.4.

[11]PTV Planung Transport Verkehr AG. VISSIM User Manual-VAP Version 2.14 APPENDIX A: ADD-ON VAP [R]. PTV Corporation, 2003. 